\title{
Effect of Rajyogi lifestyle in controlling and managing blood glycemic levels in participants in Madhur Madhumeh camps for type 2 diabetes patients
}

\author{
Valsalan Nair ${ }^{1, *}$, Prathibha Nair ${ }^{2}$, Brajesh Singhal $^{3}$, Banarsilal Sah ${ }^{4}$, Abhyudaya Verma $^{5}$ \\ ${ }^{\mathbf{1}}$ Project Director, ${ }^{\mathbf{2}}$ Coordinator, ${ }^{\mathbf{1}, 2}$ Madhur Madhumeh Project, Medical Wing, Rajyoga Education \& Research Foundation, \\ Brahma Kumaris Shantivan, Rajasthan, ${ }^{3}$ Senior Consultant, Navjeevan Hospital, Gwalior, Madhya Pradesh, ${ }^{4}$ Director, Ever \\ Healthy Hospital, Brahma Kumaris Shantivan, Rajasthan, ${ }^{5}$ Senior Endocrinologist, SEWA, Indore, Madhya Pradesh, India
}

\section{*Corresponding Author:}

Email: bkdrnair@gmail.com

\begin{abstract}
Objective: The effect of spiritually augmented healthy lifestyle intervention on clinical risk factors in patients with type 2 diabetes (T2DM) is still unclear. The aim and objective of this study was to assess the beneficial effects of a user-friendly, noninvasive spiritually augmented healthy lifestyle intervention to improve blood glucose levels in patients with type 2 diabetes within a reasonable time frame.

Materials and Methods: A prospective study was conducted over a period of one year on 552 subjects who attended Madhur Madhumeh camps in the age group of 30 years to 82 years (mean 57.82, SD 10.38) with T2DM of at least 6 months duration and on diabetic diet and oral hypoglycemic agents and/or insulin (treatment). All the participants were trained by Rajyoga experts. In all the participants pre-camp fasting (FBS) and random (RBS) blood glucose levels were taken and further fasting and random (after $3 \frac{1}{2}$ hours of lunch) blood glucose levels were estimated in regular intervals during the camp to obtain 3 readings each of fasting and random blood glucose levels.

Results: Comparing with standard care alone, Rajyogi lifestyle resulted in significant reduction in blood glucose levels within 24 hrs to 48 hours even after their high fasting (Max.455, 141 participants above $200 \mathrm{mg} / \mathrm{dL}$ ) and random (Max.700, 104 participants above $300 \mathrm{mg} / \mathrm{dL}$ ) blood glucose levels. The reduction in FBS (mean 28.24, p < 0.001) and RBS (mean 69.25, p < 0.001 ) at the end of the camp is highly significant in both male (FBS mean reduction 31.1, RBS mean reduction 74.69) and female (FBS mean reduction 24.73, RBS mean reduction 62.57) when compared with corresponding values at the beginning of the camp.

Conclusions: The study finds that Rajyogi lifestyle intervention showed significant benefit in reduction of blood glucose levels in T2DM patients. It is evident that Rajyogi lifestyle can be used as an adjunct therapy in effectively controlling and managing blood glycemic levels in patients with type 2 diabetes. Translating this finding into effective long term implementation requires further studies and evaluation.
\end{abstract}

Keywords: Diabetes, T2DM, Rajyoga, Yogic lifestyle, Meditation.

\section{Introduction}

The World Health Organization (WHO) has projected that the maximum increase in diabetes would occur in India. ${ }^{8}$ Considering the large population and the high prevalence of diabetes, the burden of diabetes in India could become enormous.

DM call for an increased risk of premature birth, particularly because it is linked with a greater risk of cardiovascular diseases. In addition, DM patients are at greater risk of becoming blind, of suffering from renal insufficiency and losing their lower limbs to amputation. Diabetes patients need to maintain strict glycemic control and reduce hyperglycemia, without increasing their risk for hypoglycemia. Blood glucose levels give us both measurable result of this optimization and serves as a principal feedback signal to the patient for his/her control of diabetes. ${ }^{4,5}$

Patients with DM must cope with a wide range of challenges specific not only with the disease but also to other areas of their lives, which influences their disease management and metabolic control. According to the American Association of Diabetes Educators: Psychological stress directly affects health and indirectly influences a person's motivation to keep his/her diabetes under control which can cause a deterioration in his/her ability to self-manage their disease. $^{7}$

Over a period, awareness programmers, medication and lifestyle modifications were used in different ways to control type 2 diabetes. Type 2 diabetes can often be prevented by lifestyle modification; however, successful lifestyle intervention programmers are labour intensive. ${ }^{1}$ It was found that diabetes selfmanagement is demanding and complex. Monitoring blood glucose, injecting insulin, oral medication, regular physical activities and healthy eating require a comprehensive understanding of not only diabetes but also of healthy coping skills in real life situations. Diabetes is more than a physical health condition, it has behavioral, psychological and social impacts. It demands high levels of self-efficacy, resilience, perceived control and overall empowerment of the self. ${ }^{2}$

Prospective cohort studies show that "low-risk lifestyle behavior" can slow onset of type 2 diabetes in both adult men and women (level II evidence). ${ }^{3,4}$ The Diabetes Prevention Program (DPP) (level I evidence) in the United States examined strategies to evaluate the safety and efficacy of lifestyle and pharmacologic 
interventions to delay or prevent development of type 2 diabetes in a diverse, high-risk population (with impaired glucose tolerance). ${ }^{5}$

There is a relatively long time gap between exposure to a risk factor and development of diabetes to its chronic stage. Therefore, an early screening for the detection of risk factors will help to reduce the mortality, morbidity, and disability. Lifestyle modification is the chief non-pharmacological method for prevention and control of diabetes and its risk factors. The importance of spirituality and lifestyle factors as contributors to and for fostering individual well-being and for preserving and optimizing cognitive function has been established time and again by various researchers all over the world. ${ }^{6}$

Lifestyle modification involves altering long-term habits, typically of eating or physical activity and maintaining the new behavior for months or years. Lifestyle modification can be used to treat a range of diseases, including diabetes. Unhealthy behaviors place many persons at risk for a variety of diseases. Lifestyle practices account for more than $40 \%$ of the differences in health among individuals (Satcher \& Higginbotham, 2008).

Mindfulness skills learnt as part of meditation could possibly help in reducing negative perseverative cognitions and thus preventing the onset of anxiety, mood disorders known to complicate medical management and self-care in people with chronic diseases. Meditation and mindfulness skills help to improve sleep, greater relaxation, and more-accepting approaches to illness and illness experience. ${ }^{7}$

Yoga and meditation were extensively studied for their beneficial effects on human health. ${ }^{8,9}$ The effect of yogic lifestyle intervention on type 2 diabetes is still unclear. For years, exercise has been considered as a cornerstone of diabetes management, along with diet and medication. Considering the fact that a large percentage of people in India are inactive with fewer than $10 \%$ engaging in recreational physical activity, ${ }^{10}$ there is an urgent need of introduction of a time tested, user friendly and non-invasive intervention as an adjunct therapy to control and manage diabetes.

Madhur Madhumeh Project for type 2 diabetes and pre-diabetes patients is a unique holistic health education program developed by Medical Wing of Rajyoga Education \& Research Foundation in association with Prajapita Brahma Kumaris Ishwariya Vishwa Vidyalaya, Mount Abu. The Madhur Madhumeh camps were based on Rajyogi lifestyle, a spiritually augmented healthy lifestyle as taught by the Brahma Kumaris for the past eight decades and practiced by lakhs of people across the world. Rajyogi lifestyle as taught by the Brahma Kumaris includes Rajyoga Meditation, Positive Thinking Classes, Satwik Diet (balanced vegetarian diet excluding onion and garlic that is prepared and taken in loving, peaceful remembrance of the Supreme and offered to the
Supreme before taking) and Practice of Soul Consciousness.

Kiran et al (2005) ${ }^{11}$ defines Brahma Kumaris Rajyoga as a method of autogenic relaxation with spiritual link, providing training in realization of the true self, contemplation on divine "Supreme Being" and a dialogue with the Supreme. Autogenic relaxation is defined as a method for influencing one's autonomic nervous system. ${ }^{12}$ Kiran further refines the definition of Rajyoga meditation as a Spiritual based relaxation therapy and Kiran et al (2009) $)^{13}$ define Rajyoga meditation as a method of meditation based on spirituality involving realization of true conscious of self, realization of attributes like peace, purity, happiness, bliss, love, power and knowledge and linking the self with "Supreme Being" by contemplating on divine Supreme.

Gupta (2006) ${ }^{14}$ defines Brahma Kumaris Rajyoga meditation is a science and art of harmonizing spiritual energy (energy of soul), mental energy (energy of mind) and physical energy (energy of physical body), through the connection with ultimate source of spiritual energy i.e. Supreme Soul for enjoying ever healthy, ever wealthy and ever happy life. Patel and Giorgio $(2005)^{15}$ insist that Rajyoga meditation is taught within the context of a lived faith complete with daily teachings, practices and lifestyle observances.

Unlike other forms of yogic methods, Rajyoga and Rajyogi lifestyle follows the natural flow of mind which is to be constantly engaged in thought. It teaches that the inner change has to occur at the level of mind to eliminate unwanted behaviors and to achieve a state of peace of mind. Concentration is not on the object or mantra but on the positive qualities of the inner self or soul - that part of mind that includes realization, feeling, will, thought, consciousness, intellect and judgement (BK Jayanti, 2000). ${ }^{16}$ One of the key variables that arise in Rajyogi lifestyle practitioners is that they experience peace of mind. ${ }^{17}$

Different forms of emotional stress increases the risk of development of type 2 diabetes. In several papers, childhood trauma and life events have been linked with onset of type 2 diabetes. It is a fact that stressful life events has an adverse effect on health. The number and duration of stressful life events increases the risk of developing diseases. An optimistic approach will help in an early recovery from trauma and will also help better immune functions. Madhur Madhumeh Project aims at bringing back peace of mind, inner strength, skills and talents to improve communications, clarify perceptions, helps to connect to own inner self and to the Supreme to live a life that is simple, natural and worth living.

All the above modern day problems were being addressed in the Madhur Madhumeh camps through three principles, intrinsic to the camp, which underlie the program ethos. 
i) Health in Your Hands - Empowering the Self: To live healthy, one has to develop not only physical immunity but also mental immunity. So, empowering individuals to take care of stress and emotions is equally important as making them aware of following a healthy diet and regular exercise. The word healthy means heal thy (self). Healing the self will happen only when individuals are empowered with holistic health concepts and the knowledge to control and manage physical, psychological and conventional risk factors.

Our role in this material world, our material possessions and consciousness of body are mortal and results in instability and insecurity. The individual expresses this as anger, anxiety, depression, isolation, chronic stress and type A behavior. The knowledge of the self as a soul, a source of immortal energy, leads to stability and security which in turn bring peace, love and happiness which are very much essential to lead a healthy lifestyle.

ii) Multi-dimensionality: It is a fact that noncommunicable diseases are growing alarmingly despite all the medical advancements. We strongly felt that we still have to look beyond conventional way of looking at human being as a physical body (one dimension). Madhur Madhumeh follows a 3D healthcare model that considers soul and mind in addition to the body as essential components of healing. In this model, health is a dynamic process and the harmony of spiritual energy, mental energy and physical energy is necessary for one to be completely healthy.

iii) Biological clock: A biological clock is a molecular mechanism that results in a circadian rhythm in a living being. Our sleep and waking patterns, digestion, blood pressure and functioning of the kidneys, are effectively adapted to each other and activated at the correct time of the day by the biological clock. As this clock is also there in the cells of our body, depending on the time of day, our body is more sensitive or less sensitive to certain substances/circumstances.

By sustaining the above three principles in our daily living, we will be able to restore balance with our inner and outer worlds. Finding inner peace and replenishing spiritual power can be satisfied by following a healthy lifestyle. The Madhur Madhumeh program provided a learning environment for diabetes patients to acquire necessary social and life skills to control and manage diabetes and to live a healthy living. Madhur Madhumeh program is based on three major components namely Relax and Relieve, Satwik Diet and Physical Activities.

i) Relax and Relieve: Once internal and external pressures exceed our inner strength, stress develops, resulting in over activity of sympathetic system and release of stress hormones thereby paving way to mental and physical dis-ease. Deep relaxation helps the individual to feel peace within and focus their attention and prioritize their needs. Inner strength or coping skills can be enhanced by Rajyoga. Raj yoga word has been derived from Raja which means king and Yoga which means union of energies of the Self and the Supreme. Rajyoga harmonizes spiritual, mental and physical energy, thereby increasing inner strength to live a stress-free and healthy life. It enhances individual's power of determination to manage and practice positive thoughts, emotions, attitudes, memories and cling to healthy diet, exercise, sleep, medication and cessation of substance of abuse.

Expert Rajyoga teachers from Rajyoga Centers of Prajapita Brahma Kumaris Ishwariya Vishwa Vidyalaya (HQ: Mount Abu, Rajasthan, India), taught Rajyoga in three different stages.

a) Stage-I: Self-Empowerment: We are surrounded by the mess of material world. We need to change our perception from physical cognizance to spiritual cognizance, seeing ourselves as a soul or spiritual consciousness that is beyond form. The natural state of the soul, its internal strength is expressed in the form of love, confidence, courage, and lots of different positive qualities. Awakening on own soul-energy, one learn to be uninfluenced, to be oneself, safe and protected in that pure energy. You also start feeling your own soulenergy. Then the whole process is to express that energy in daily life, in relationships.

b) Stage-II: Opening and healing the mind: In this stage, one learn to understand the role of non-physical factors like depression, anger, cynicism, hostility, ego, jealousy, hurry, worry, anxiety, fear, isolation, lack of social and emotional support, job and family stress etc., in the development of lifestyle diseases, as these negative traits are acquired from the environment and are not part of the original self. The training to inculcate positive mental energy enhances one's willpower to adhere to healthy lifestyle.

c) Stage-III: Cleansing by Rajyoga meditation: When one allows silence to enter the mind, one comes to know who he/she is, deep down inside. One begins to understand his/her motivations. One of the outstanding edges of correct consciousness could be a relative freedom from the wants and dependencies that unremarkably govern our lives. The participants are supposed to be focusing the inner, radiant spiritual energy on various organs of the body at this stage. This part of the session is to discipline the mind with love and compassion. It needs to be guided onto the right path. It needs to be controlled and focused and guided in the right direction, thus creating a suitable 'chain of thought'. A guided commentary was provided to relax the body and guide the mind in a positive direction and liberate the individual from all the karmic bondage (bondages created through various actions in life). By acquiring the power to forgive and forget, the individual escapes any ill feelings that arises normally and be encouraged to maintain a self-conscious mental state even while engaged in day-to-day activities.

ii) Satwik Diet: Satwik Diet is a balanced vegetarian diet excluding onion and garlic that is prepared and 
taken in loving, peaceful remembrance of the Supreme and offered to the Supreme before taking. A vegetarian diet benefits in spiritual development by helping develop the clarity, concentration and subtle focus.

a) Low carb, high fiber, pure vegetarian diet with minimal fat: The essential components of a healthy diet program are low carb, high fiber, pure vegetarian diet with minimal fat to be properly chewed in inner self-conscious state according to biological clock. The camp diet consisted of one portion of grated vegetables (vegetable salad) for both break-fast and lunch, one portion of steamed sprouted grams (only for breakfast), one portion of carbohydrate, one portion of boiled vegetables and/or one portion of beans.

b) Think local, eat local: Serving staple diet is one of the attractions of Madhur Madhumeh Camps. Food is prepared with local, seasonal, available food items in local taste but in a healthy way with minimum or no added fat.

c) Animal Products, Chilies and other Stimulants -

Big No: The participants are advised to avoid animal products till the time they are in control of their diseased condition as a supportive measure for early healing. No animal products like meat, fish, eggs, milk, curd, butter or ghee were served. Black pepper, long pepper and ginger were used in place of chilies. No stimulants like tea, coffee, onion and garlic were served. Instead of processed salt and oil, natural sea salt/rock salt and virgin vegetable oil (extracted through cold processing) were used. Root vegetables like potatoes were not recommended till the time the diseased condition comes under control, but carrots and beets were used in salads and vegetables.

iii) Physical Activity: The challenges related to blood glucose management vary with diabetes type, activity type, and presence of diabetes-related complications. Physical activity and exercise recommendations, therefore, were tailored to meet the specific needs of each individual. According to the health condition and taste of the participants, exercises like sun stroll, yoga, body patting, skipping rope, indoor and outdoor games etc. were recommended.

\section{Materials and Methods}

Medical Wing of Rajyoga Education and Research Foundation was organizing three days non-residential unique holistic health education camps for type 2 diabetes and pre-diabetes patients under the banner "Madhur Madhumeh" for the past one year. The data for the study was collected from Madhur Madhumeh camps organized in Kuppam and Vizianagaram (Andra Pradesh), Guwahati (Assam), Chhajjupur (Delhi), Chhote Udepur (Gujarat), Asandh, Bahal and Digawa Mandi (Haryana), Ernakulam, Kottayam, Thiruvalla and Thrissur (Kerala), Amaravati, Pune and Satara (Maharashtra), Balaghat, Gwalior, Indore, Jabalpur, Morena and Rewa (Madhya Pradesh), Jaipur, Jodhpur and Nagaur (Rajasthan) and Lucknow (UP).
The camp based on Rajyogi lifestyle as taught by the Brahma Kumaris, has got a standard schedule. As per the standard schedule, participants were requested to report at $4 \mathrm{pm}$ on Day 0 , the inauguration day. A standardized Registration Proforma was administered by trained interviewers on adult T2DM patients of at least 6 months duration and on diabetic diet and oral hypoglycemic agents and/or insulin. Random blood glucose levels (after $3 \frac{1}{2}$ hours of lunch) were also recorded on Day 0 and these were taken as the base random blood glucose value (pre-camp). The patients were briefed about the camp schedule and asked to report by 7 am on next day (Day 1) for the camp without consuming any food or beverage (other than plain water) from midnight until their blood sample were given for testing in the camp. Fasting blood glucose levels were recorded on Day 1 and these were taken as the base fasting blood glucose values (precamp). In addition to this, fasting blood glucose levels were recorded every day from Day 2 to Day 3 and random blood glucose levels (after $3 \frac{1}{2}$ hours of lunch) were recorded for Day 1 and Day 2 to obtain 3 readings each of fasting and random blood glucose levels.

During the camp, no changes were made in medication, staple diet and exercise of the participants except encouraging the participants to follow a healthy diet plan that was best suitable for their present health condition without any restriction on quantity of food that they were taking. Their healthy diet plan included, vegetable salad, seasonal green leafy vegetable chutney, steamed sprouted green grams with added salt for taste and their staple breakfast dish for the breakfast; vegetable salad, seasonal green leafy vegetable chutney, boiled mix local seasonal vegetables, local seasonal vegetables with gravy, beans curry and their staple lunch (rice/chapatis) for lunch. Seasonal fruits (150 gms) were served after 2 hours of breakfast and a mix vegetable soup was served after 2 hours of lunch.

The before breakfast session on Day 1 was designed as ice-breaking session. The first session after the breakfast was aimed at bringing back peace of mind and inner strength. The second session after breakfast was aimed to help participants to connect to their inner self. Post lunch sessions on Day1 helped participants to understand the Supreme Consciousness and to connect with the Supreme. Of the five sessions in Day1, three were interactive sessions and two were of deep experiential. Day 2 started with a pre-breakfast session, sharing healthy diet tips, taking into account local taste and flavour. This was followed by sessions to understand the deep principles of law of justice and to release negative traits that were acquired over a period of time. While first two sessions before lunch were interactive in nature, third session was a deep meditative experiential session. There was also a post lunch meditation session without any guided commentary. The third day sessions were designed to understand how the powers acquired through Rajyoga 
can be used to increase inner strength to live a stressfree and healthy life.

Data was collected locally but managed centrally at Medical Wing Research Office and was analysed by independent statistician using software. Paired t-test was done to compare between two groups. Significance was considered at $\mathrm{p}<0.001$ levels for all the parameters.

\section{Results and Discussions}

The present study of 552 persons with type 2 diabetes and pre-diabetes in the age group of 30 years and above, $53.47 \%$ urban and rest rural from 10 Indian States, thus having a pan India representation. 470 persons $(85.14 \%)$ attended the camps fully wherein 259 $(55.11 \%)$ were male and $211(44.89 \%)$ were female. The median dropout rate of $12.5 \%$ after 48 hours of the camps show better adherence rate and acceptability of the camps.

Fasting blood glucose level of 101 participants were $>200$ but $<=300$ and 37 participants were more than 300 whereas random blood glucose level of 127 participants were $>200$ but $<=300$ and 103 participants were more than 300. Average fasting blood glucose level came down to 163.87 from 202.78 and average random blood glucose level came down to 156.98 from 229.54 in 48 hours of persons who attended camps fully.

Median fasting blood glucose level of male and female participants showed a drop of $15 \%$ and $10.65 \%$ in 24 hours and $17.49 \%$ and $13.73 \%$ respectively in 48 hours whereas the random blood glucose levels came down by $27.42 \%$ and $24.49 \%$ in male and $32.94 \%$ and $28.60 \%$ respectively in female participants.

Table 1: Result of fasting blood glucose level - Madhur Madhumeh camp (t-test)

\begin{tabular}{|c|c|c|c|c|}
\hline Group & $\mathbf{N}$ & Mean & SD & p Value \\
\hline Before Camp & 470 & 178.856 & 72.559 & \multirow{3}{*}{$<0.001$} \\
\hline After 24 hours in camp & 470 & 155.548 & 55.629 & \\
\hline After 48 hours in camp & 470 & 150.612 & 51.525 & \\
\hline
\end{tabular}

Table 2: Result of random blood glucose level (after 31/2 hours of lunch) - Madhur Madhumeh camp (t-test)

\begin{tabular}{|l|c|c|c|c|}
\hline \multicolumn{1}{|c|}{ Group } & N & Mean & SD & p Value \\
\cline { 1 - 4 } Before Camp & 470 & 223.166 & 103.116 & \multirow{3}{*}{$<0.001$} \\
\cline { 1 - 4 } After 24 hours in camp & 470 & 164.852 & 74.274 & \\
\hline After 48 hours in camp & 470 & 153.919 & 70.161 & \\
\hline
\end{tabular}

\section{ADHERERANCE RATE}

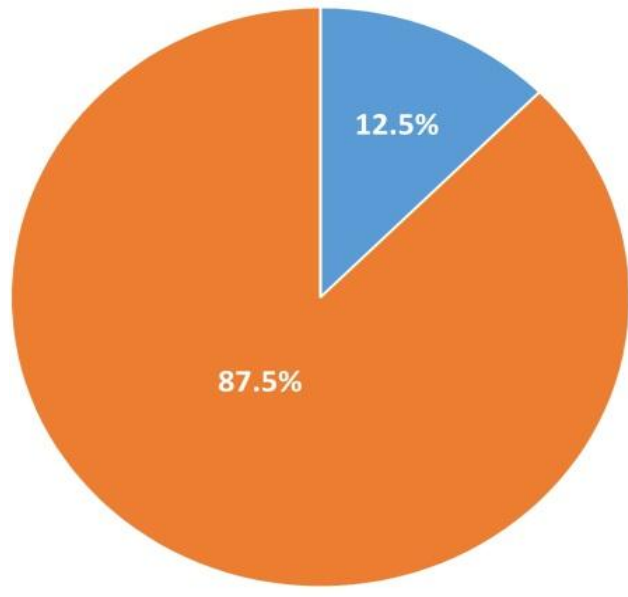

DROP OUT = FULLY ATTENDED

Fig. 1: Diabetes - adherence

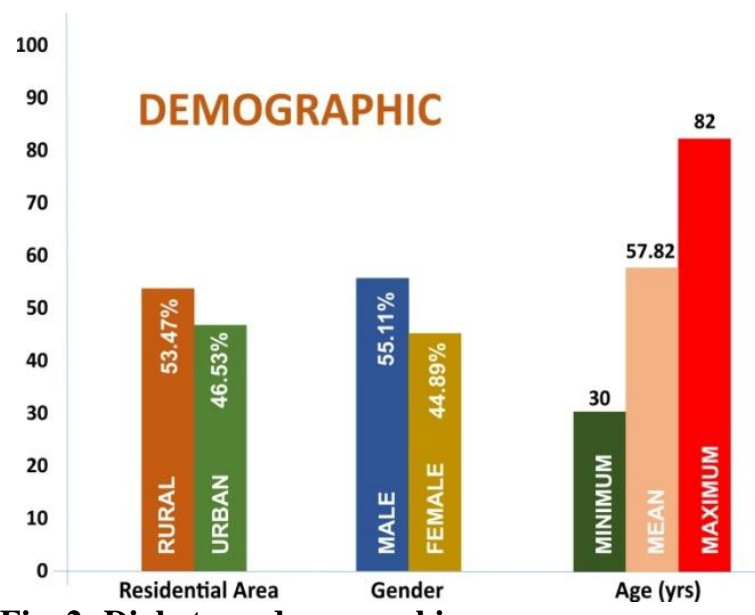

Fig. 2: Diabetes - demographic 


\section{COMPARISON OF GLYCEMIC LEVELS}

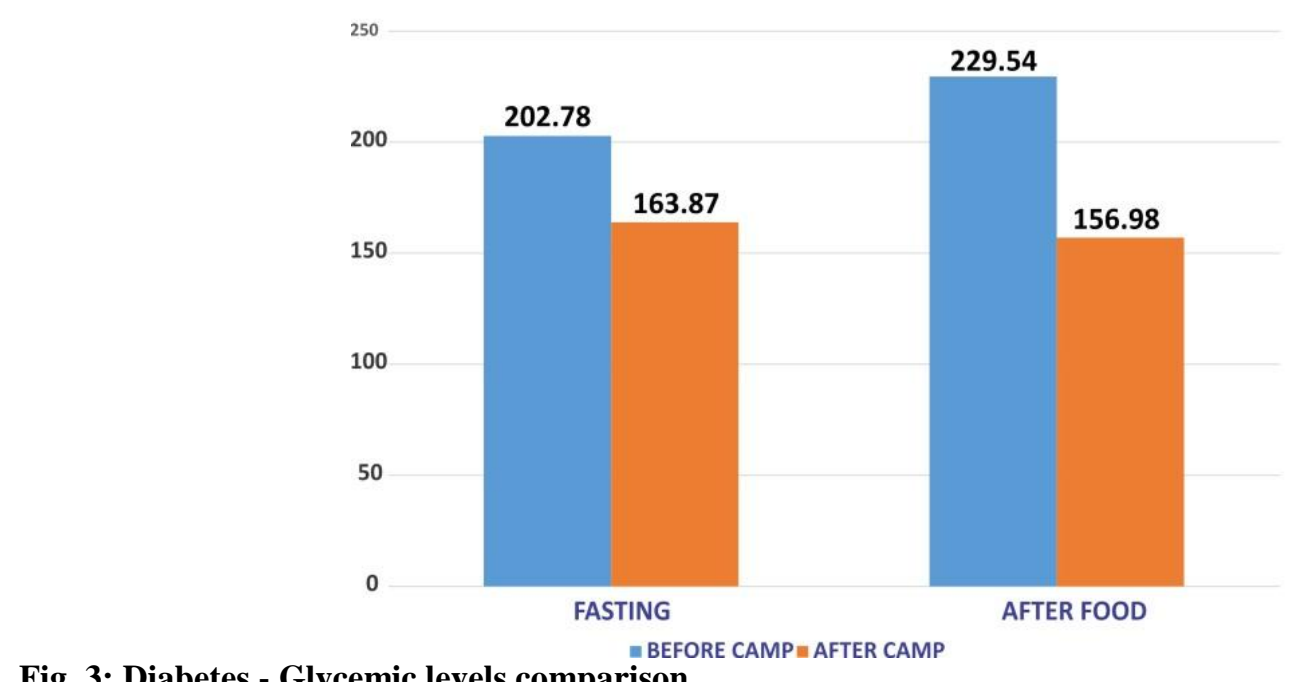

Fig. 3: Diabetes - Glycemic levels comparison

\section{GLYCEMIC LEVELS}

BEFORE CAMP

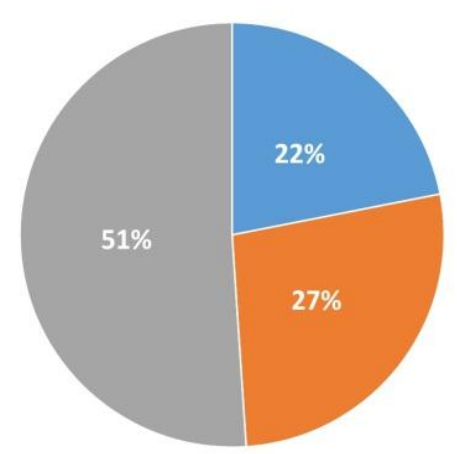

ABOVE $300=>200$ BUT $<=300=$ UPTO 200

Fig. 4: Diabetes - glycemic levels

\section{Conclusion}

It is proved that empowerment of individual patients to manage and control their diseases through healthy lifestyle intervention improves blood glucose levels. The findings of this study conclude that Rajyogi lifestyle is effective in control and management of blood glycemic levels in type 2 diabetes patients within a reasonable time frame. The reasonably low dropout rate $(12.57 \%)$ in our study also indicates that subjects with impaired glucose tolerance are willing and able to participate in a demanding intervention program if it is made available to them. We would like to see the improvement with sustainability of the results over a period of time. To analyze the rationale behind this and to analyze the long term effect of Rajyogi lifestyle, a more detailed scientific study is being planned and

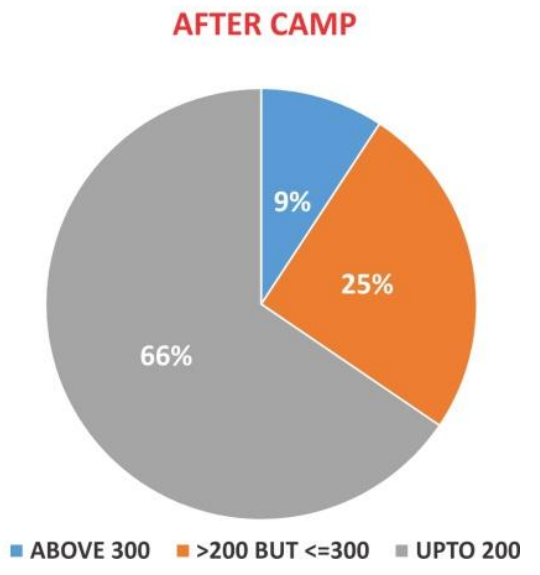

various factors like ease of adaptability, consistency in lowering of blood glucose levels etc. will be analyzed.

\section{References}

1. Ramachandran A, Snehalatha C, Ram J, Selvam S, Simon M, Nanditha A, Shetty AS, Godsland IF, Chaturvedi N, Majeed A, Oliver N, Toumazou C, Alberti KG, Johnston DG. Effectiveness of mobile phone messaging in prevention of type 2 diabetes by lifestyle modification in men in India: a prospective, parallel-group, randomised controlled trial.

2. Medical Wing of Rajyoga Education \& Research Foundation (2017), Sweet Diabetes.

3. Hu FB, Manson JE, Stampfer MJ, Colditz G, Liu S, Solomon CG, et al. Diet, life-style, and the risk of type 2 diabetes mellitus in women. $N$ Engl $\mathrm{J}$ Med 2001;345(11):790-7.25. 
4. Manson JE, Nathan DM, Krolewski AS, Stampfer MJ, Willett WC, Hennekens CH. A prospective study of exercise and incidence of diabetes among US male physicians. JAMA 1992;268(1):63-7.

5. Diabetes Prevention Program Research Group. Reduction in the incidence of Type 2 diabetes with lifestyle intervention or metformin. $N$ Engl J Med 2002;346(6):393-403.

6. King, H., Aubert, R., and Herman, W. Global burden of diabetes, 1995-2025: prevalence, numerical estimates and projections. Diabetes Care. 1998;21:1414-1431

7. Chris Keyworth, Jasmin Knopp, Kate Roughley, Chris Dickens, Stuart Bold \& Peter Coventry. A MixedMethods Pilot Study of the Acceptability and Effectiveness of a Brief Meditation and Mindfulness Intervention for People with Diabetes and Coronary Heart Disease. Journal of Behavioral Medicine Volume 40, 2014 - Issue 2 Pages 53-64, 06 Sep 2013. https://doi.org/10.1080/08964289.2013.834865

8. Anand BK. Yoga and medical science. Indian J Physiol Pharmacol 35(2):1991; 84-87.

9. Cooper MJ, Aygen MM. A relaxation technique in the management of hypercholesterolemia. J Human Stress 1979 Dec;5(4): 24-27.

10. Ranjit M Anjana, RajendraPradeepa, Ashok K Das, Mohan Deepa, Anil Bhansali, Shashank R Joshi, Prashant P Joshi, Vinay K Dhandhania, Paturi V Rao, Vasudevan
Sudha, Radhakrishnan Subashini, Ranjit Unnikrishnan, Sri V Madhu, Tanvir Kaur, Viswanathan Mohan, and Deepak K Shukla, for the ICMR- INDIAB Collaborative Study Group. Physical activity and inactivity patterns in India - results from the ICMR-INDIAB study (Phase-1) [ICMR-INDIAB-5]. Int J BehavNutr Phys Act. 2014;11:26.

11. Kiran U, Behrai M, Venugopal P, Vivekanandhan S, Pandey RM. The Effect of Autogenic Relaxation on Chronic Tension Headache and in modulating cortisol response. Indian J. Anaesth 2005;49(6):474-478.

12. http://en.wikipedia.org/wiki/Autogenic_training.

13. Kiran U, Makhija N, Malik V, Gharde P. Relaxation Therapies in Pain Management. Indian J PAIN 2009;23:218-225.

14. Gupta S Kr. Soul-Mind-Body Medicine for Healthy Happy Living: for Prevention of Angina and Heart Attacks. World Congress on Clinical and Preventative Cardiology 22 - 24 Sept 2006 Souvenir Program.

15. Patel N, Giorgio B, Meditation in a lived faith context as therapeutic intervention for substance abuse, dependence and addiction: an empirical study. Archives of Indian Psychiatry 2005;7:(1) 52-56.

16. B.K. Jayanti (2000), Practical Meditation, Health Communication Inc., Florida.

17. O’Donnell, K. (2000) Raj Yoga: New Beginnings, Eternity Inc., Sydney, Australia. 